\title{
Positive selection and gene duplications in tumour suppressor genes reveal clues about
} how cetaceans resist cancer

Daniela Tejada-Martinez ${ }^{1,2,3,4}$, João Pedro de Magalhães ${ }^{3}$ and Juan C. Opazo ${ }^{2,5,6}$

${ }^{1}$ Programa de Doctorado en Ciencias mención Ecología y Evolución, Facultad de Ciencias, Universidad Austral de Chile, Valdivia, Chile.

${ }^{2}$ Instituto de Ciencias Ambientales y Evolutivas, Facultad de Ciencias, Universidad Austral de Chile, Valdivia, Chile.

${ }^{3}$ Integrative Genomics of Ageing Group, Institute of Ageing and Chronic Disease, University of Liverpool, Liverpool L7 8TX, UK.

${ }^{4}$ Department of Biochemistry and Molecular Biology, Thomas Jefferson University, Philadelphia, PA, 19107, USA

${ }^{5}$ Millennium Nucleus of Ion Channels-Associated Diseases (MiNICAD), Valdivia, Chile

${ }^{6}$ Integrative Biology Group, Universidad Austral de Chile, Valdivia, Chile

Corresponding authors: dtejadamartinez@gmail.com; jp@senescence.info;

jopazo@gmail.com 


\begin{abstract}
Cetaceans are the longest-living species of mammals and the largest in the history of the planet. They have developed mechanisms against diseases such cancer, although the underlying molecular bases of these remain unknown. The goal of this study was to investigate the role of natural selection in the evolution of 1077 tumour suppressor genes (TSGs) in cetaceans. We used a comparative genomic approach to analyse two sources of molecular variation in the form of $\mathrm{dN} / \mathrm{dS}$ rates and gene copy number variation. We found a signal of positive selection in the ancestor of cetaceans within the CXCR2 gene, an important regulator of DNA-damage, tumour dissemination, and immune system. Further, in the ancestor of baleen whales, we found six genes exhibiting positive selection relating to such diseases as breast carcinoma, lung neoplasm (ADAMTS8) and leukaemia (ANXA1). The TSG turnover rate (gene gain and loss) was almost 2.4-fold higher in cetaceans as compared to other mammals, and noticeably even faster in baleen whales. The molecular variants in TSGs found in baleen whales, combined with the faster gene turnover rate, could have favoured the evolution of their particular traits of anti-cancer resistance, gigantism and longevity. Additionally, we report 71 genes with duplications, of which 11 genes are linked to longevity (e.g. NOTCH3 and SIK1) and are important regulators of senescence, cell proliferation and metabolism. Overall, these results provide evolutionary evidence that natural selection in tumour suppressor genes could act on species with large body sizes and extended life span, providing novel insights into the genetic basis of disease resistance.
\end{abstract}

Keywords: Comparative genomics, cancer evolution, longevity, whales. 


\section{Background}

Why do not larger organisms always have a higher risk of developing cancer than smaller ones? This is an unresolved question that was first observed by Richard Peto in 1977 [1] which later became known as "Peto's paradox" [2,3]. This paradox is based on the observation that the risk of developing cancer should increase with the number of cells. The higher number of cells the greater the number of cell divisions and the higher the probability of DNA damage and the transformation of a normal cell into a cancerous one $[4,5]$. Accordingly, we should expect a higher probability of developing cancer in larger and longer-lived organisms than smaller ones, although this does not occur [6-10]. Several hypotheses have been proposed to explain the different evolutionary strategies that have arisen to fight cancer [11], including specific adaptations to the environment, fitness, DNA damage responses, tissue microenvironments, and cellular senescence $[12,13]$. Recently, the availability of the genomes of a wide variety of species has prompted the search for genes that could explain the differences arising in cancer incidence between species [14]. In particular, understanding the molecular basis of the anticancer mechanisms in large and long-lived species may shed light on several fundamental questions in medicine.

How organisms reach and maintain larger body sizes and/or achieve increased longevity has puzzled scientists for decades $[15,16]$. For example, the naked mole rat (Heterocephalus glaber) has an average body mass of $35 \mathrm{~g}$ and a lifespan of 35 years [17]. Long-term studies suggest that their mortality rate [18] and the risk of developing cancer does not seem to increase with age [19]. In this species, special mechanisms like early contact inhibition would be, in part, responsible for their extended lifespan [20,21]. Among vertebrates, other anticancer-ageing mechanisms have been described which could potentially explain the maintenance of large body sizes and/or increased longevity [11]. For example, in the little brown bat (Myotis lucifugus) telomere dynamics and changes in the genes associated with growth factors are related to repair mechanisms that prevent DNA damage associated with ageing $[22,23]$. In the yellowmouth rockfish (Sebastes reedi), there are no signs of aging in replicative senescence [24], while in the African elephant (Loxodonta africana) it is explained as a variation arising in gene copy number in TP53 and LIF genes [25]. Molecular variation in tumour suppressor genes could work in the manner of a compensatory mechanism, providing additional and novel ways to avoid or prevent cancer [4]. However, the evolutionary history of tumour suppressor genes remains unknown. 
Cetaceans evolved from a terrestrial ancestor and re-entered into the oceans around 50 million years ago [26]. Their adaptation to an aquatic lifestyle required many morphological, physiological, and behavioural changes. The sub-order Cetacea comprises two main lineages; namely Odontoceti (or toothed whales) and Mysticeti (or baleen whales) [26]. Baleen whales and toothed whales display a vast variation in terms of body mass and maximum lifespan, ranging from 17 years for the Pygmy sperm whale (Kogia breviceps) to 211 years in the bowhead whale (Balaena mysticetus); and from $50 \mathrm{~kg}$ for Maui's dolphin (Cephalorhynchus hectori maui) to 175 tons for the blue whale (Balaenoptera musculus) [17]. Because there are species that live over a hundred years, they are exposed for a much longer period to the appearance of harmful mutations, pathogens, or diseases. Thus, it is expected that cetaceans developed improvements in their immune systems, DNA repair mechanisms and other biological processes which could act to favour greater longevity and body mass [27,28]. Further, recent investigations have found a signal for the positive selection of those genes which could be related to the observed variations in terms of body size [27]. However, although in mammals the body of evidence suggests that tumour suppressor genes play a key role in the evolution of body size and longevity by decreasing the incidence of diseases such as cancer [15,29], it still remains an open question as to which molecular variants in cetaceans are responsible for the lower observed incidence of cancer [30-32].

The goal of this study is to investigate the evolution of tumour suppressor genes in cetaceans by evaluating two forms of molecular variation, namely $\mathrm{d}_{\mathrm{N}} / \mathrm{d}_{\mathrm{S}}$ and gene copy number. We report a signal of positive selection in those genes which are involved in the control of cancer onset and progression. The turnover rate of tumour suppressor genes was faster in cetaceans relative to other mammals. We report 71 genes exhibiting duplications in one or more species of cetaceans which are known to be involved in multiple human disorders and ageing. This approach highlights how studying the role of natural selection within genes associated with human health could lead to advances in our understanding of the genetic basis of disease resistance.

\section{Materials and Methods}

\section{Relationship between body mass and longevity in cetaceans}

We used the AnAge database to evaluate if there exist differences between the longevity and body mass in cetaceans relative to other mammals. The Caper R-package was used to make a linear regression through the Phylogenetic generalized least squares (PGLS) [33] models. 
PGLS assumes a multivariate Brownian motion process and consider the phylogenetic relationships among species. The phylogenetic tree was obtained from Uyeda et al. (2017) [34].

\section{DNA sequences and taxonomic sampling}

To study the evolution of tumour suppressor genes (TSGs) in cetaceans we implemented a phylogenetic design including 15 mammalian species. Our taxonomic sampling included five Odontocetes (bottlenose dolphin, Tursiops truncatus; orca, Orcinus orca; beluga, Delphinapterus leucas; Yangtze river dolphin, Lipotes vexillifer; the sperm whale, Physeter catodon); two Mysticetes (common minke whale, Balaenoptera acutorostrata; bowhead whale, Balaena mysticetus); five other members of the superorder Laurasiatheria (cow, Bos taurus; pig, Sus scrofa; dog, Canis familiaris; horse, Equus caballus; microbat Myotis lucifugus); two Euarchontoglires (human, Homo sapiens; mouse, Mus musculus); and one Atlantogenata (African elephant, Loxodonta africana). The coding sequences for each species were downloaded from Ensembl v.96 (http://www.ensembl.org), NCBI database [35] and the Bowhead whale genome project (http://www.bowhead-whale.org/) (supplementary table 1). To remove the low-quality records, sequences were clustered using CD-HITest v.4.6 [36] with a sequence identity threshold of $90 \%$ and an alignment coverage control of $80 \%$. After that, the longest open reading frame was kept using TransDecoder LongOrfs and TransDecoder-predicted in TransDecoder v3.0.1 (https://github.com/TransDecoder/TransDecoder/).

\section{Homology inference}

We inferred homologous relationships between the 1077 tumour suppressor genes described for humans, which are available from publically accessible databases (Tumor Suppressor Gene Database, https://bioinfo.uth.edu/TSGene/ and the Tumor Associate Gene, http://www.binfo.ncku.edu.tw/TAG/GeneDoc.php) (supplementary table 2), and the other 14 species included in our study using the program OMA standalone v.2.3.1 [37]. OMA standalone is a graph-based method that infers homology using strict pairwise sequence comparisons "all-against-all" based on evolutionary distances. It also has a survey of the relationships between species, which for this study were included a priori. We inferred two types of groupings: 1) OMA Groups (OG), containing the sets of orthologous genes, and 2) Hierarchical Orthologous Groups (HOGs). Briefly, tHOGs are those genes that have descended from a single ancestral gene within a species against which gene duplications and 
losses can be contrasted. This algorithm has previously shown high precision, minimising the errors in orthology assignment in comparison with other homology inference methods $[38,39]$. Using these HOGs groups, the number of copies of tumour suppressor genes was obtained on a per species basis. We used a home-made script as an extra filter so as not to overestimate the number of copies of TSG. For each HOG we filtered the longest transcript given the same GeneID (supplementary file 5).

The amino acid sequences were aligned using the L-INS-i algorithm from MAFFT v.7 [40]. Nucleotide alignments were generated using the amino acid alignments as a template using the function pxaa2cdn in phyx [41]. Finally, to reduce the chance of false positives arising within low-quality alignment regions, we added a cleaning step using the codon.clean.msa algorithm of the rphast package [42] with the associated human tumour suppressor gene as reference sequence.

\section{Natural selection analysis}

To evaluate the role of natural selection in the evolution of tumour suppressor genes, we employed the codon-based model within a maximum likelihood framework using the program PAML v4.9 [43], as it is implemented in the ETE-toolkit with the ete-evol function [44]. We used the branch-site model to estimate the signature of positive selection with the last common ancestor of Cetacea, Mysticeti (baleen whales), and Odontoceti (toothed whales). We compared the null model, wherein the value of $\omega$ in the foreground branch was set to 1 , with the model in which the omega value was estimated from the data using the likelihood ratio tests (LRT) [45]. We applied the false discovery rate (FDR) [46] as a multiple tests correction. The genes with an FDR $<1$ were categorised with a positive selection. Finally, we used branch models to compare the dN/dS distribution of TSGs between cetaceans and other mammals. We implemented a model in which an omega value was estimated for cetaceans and another for all other branches of the tree. To estimate whether the omega distributions were significantly different, we used the Kolmogorov-Smirnov test in $\mathrm{R}$ (www.r-project.org/).

\section{Gene copy number variation analysis}

To estimate the gene turnover rate of the tumour suppressor genes we used the software CAFE, v. 4.2.1 (Computational Analysis of Gene Family Evolution) [47]. CAFE uses a stochastic birth and death model to estimate the expansions and contractions of genes using, as a frame of reference, sister group relationships arising among species relative to 
divergence times. Using this approach we can infer a maximum likelihood value for the rate of evolution $(\lambda)$ and the direction of the change in terms of the size of the gene families across different lineages with a $p<0.01$. The $p$-value describes the likelihood of the observed number of genes appearing given average rates of gain and loss. All the comparisons were calculated with a genome assembly and annotation random error, assuming that all branches in the tree share the same unique $\lambda$. The error model was calculated using the caferror.py script in CAFE [47]. The estimated error value $=0.06875$ was included in the further estimations. The family-wide $p$-value describes the likelihood of the observed number of genes arising, given average rates of gain and loss (please see supplementary files 3-4). We implemented two independent models. In the first, one $\lambda$ was estimated for cetaceans as a total group and the other for the outgroup. In the second model, we estimated four $\lambda$ values for (i) the stem cetácea; (ii) the total group of Mysticeti; (iii) the total group of Odontoceti; and (iv) the outgroup, respectively. The divergence time between species was obtained from the TimeTree database (http://www.timetree.org/) [48]. However, it should be noted that Odontoceti exhibit a polytomy, so we then re-calibrated the tree using the R packages APE and Phytools $[49,50]$ with the estimated ages and a time-calibrated phylogeny for Odontoceti [51].

\section{Enrichment analysis}

The enrichment analysis was performed using the R package WebGestaltR v0.4.4 [52]. The set of duplicated genes were tested for significant pathway associations using the hypergeometric test for Over-Representation Analysis (ORA) [53]. We selected the categories of gene ontology biological processes, diseases OMIM, and human phenotype and we considered overrepresented categories to be those with a significance level above that of an FDR of 0.01 after correction with the Benjamini-Hochberg multiple test. The enrichment analysis was made independently using as a background the human protein-coding genes and relative to the 1,077 TSGs. Finally, we overlapped the TSGs in terms of positive selection and copy number variation with the GenAge database (build 19,307 genes) [17] and CellAge Database of Cell Senescence Genes [54].

To test if the TSGs with duplications are involved in the longevity process more than would be expected by chance, we performed a gene overlap analysis using the GeneOverlap package in $\mathrm{R}$ [55]. The strength of the association was measured via the odds ratio, wherein an odds ratio $<1$ implies no association between the lists; and an odds ratio $>1$ infers a strong association. Protein coding genes from humans were used as a background (20,183 
genes). To make the comparison, we merged the list of genes from the GenAge database and the CellAge Database of Cell Senescence Genes as the "longevity associated genes" and we also employed the list of 1,045 TSGs obtained from the Hierarchical Ortho Groups (HOG) as well as the list of 72 TSGs.

\section{Results}

Relationship arising between body mass and longevity in cetaceans

We compared the relationship between longevity and body mass in both cetaceans and other mammals. The PGLS analysis (see Materials and Methods), using data obtained from the AnAge database, reveals that the relationship is significantly different across cetaceans in comparison to other mammalian species ( $p$-value: $<2.2 \mathrm{e}-16$, Residual standard error: 0.04185 on 929 degrees of freedom, multiple R-squared: 0.08159 , adjusted R-squared: 0.07862 , figure 1).

\section{Homology inference}

We performed an evolutionary analysis to understand the role of natural selection in the evolution of tumour suppressor genes in cetaceans. Employing the 1,077 TSGs described for humans, we obtained 362 OrthoGroups (OG) from the 15 mammalian species included in this study (supplementary file 1) as well as 1,044 hierarchical orthologous groups (HOG) containing two or more species (supplementary file 2).

TSGs with positive selection in cetaceans are correlated with multiple human disorders Within the 362 orthologous genes selected, we found that a signal for positive selection within 7 tumour suppressor genes (figure 2a, supplementary table 3). We report that the $C X C R 2$ gene exhibits a signature of positive selection in the ancestor of cetaceans and also in the genes DAB2, ADAMTS8, DSC3, EPHA2, TMPRSS11A, ANXA1 in the ancestor of baleen whales. The GeneCards - Diseases associated database showed that those genes with the signature of positive selection are implicated in multiple forms of cancer, such as lung neoplasm (ADAMTS8), leukemia (ANXA1), and teratocarcinoma (DAB2) as well as in immune system disorders such as congenital neutropenia syndrome (CXCR2) (table 1). In the last common ancestor of baleen whales, a lineage that includes the largest and longest-lived species of mammals, the most represented functional categories were all related to cell cycle regulation, metabolism and apoptosis. It is noteworthy to mention that several genes with the 
signature of positive selection are related to abnormalities in scalp hair and blood vessel development, phenotypes associated with the evolution of aquatic life. For the branch model, we found that the distribution of the omega values arising between groups is significantly different $(\mathrm{D}=0.32764, p$-value $<2.2 \mathrm{e}-16)$, while the kernel-smoothed density estimates of the omega values show that crown cetacea have a distribution of the mean that is greater than for the outgroup (figure $2 b$ - supplementary table 4 ).

In summary, we report signatures of positive selection arising in genes that are involved in multiple types of cancer and also in immune system disorders that could increase predisposition to cancer. Since we found more TSGs with the signature of positive selection in the ancestor of baleen whales, we suggest that these lineages could have evolved with a selection for additional anticancer/ageing mechanisms, thereby reducing the risk of cancer and thus favouring longevity and greater body mass. Studying ageing with such a comparative perspective offers an avenue that could potentially provide information about the role of natural selection in species which have found a way to avoid diseases commonly associated with ageing, such as cancer.

\section{Cetaceans have an accelerated gene turnover rate in comparison to other mammals}

The copy number variation analysis revealed that in the first model, the turnover rate of TSGs of cetaceans, as a total group, is more than two times faster $(\lambda \mathrm{c}=0.00074)$ than the rest of the tree $\left(\lambda_{0}=0.00031\right)$ (figure $\left.3 \mathrm{a}\right)$. In the second model, in which we specified four rates of gene family evolution, we found that the turnover rate values estimated for the last common ancestor of baleen whales $\left(\lambda_{\text {my }}=0.0019\right)$ was faster than for the last common ancestor of cetaceans $\left(\lambda_{C}=0.00022\right)$ or the last common ancestor of toothed whales $\left(\lambda_{\mathrm{od}}=0.00015\right)$ (figure $3 b$ ).

To gain further insights into those mechanisms pertaining to ageing and disease resistance which are associated with the tumour suppressor gene families, we identified those TSGs with specific duplications in cetaceans. According to our analyses, we identified that 71 TSGs were duplicated in one or more cetacean species (figure 4a; supplementary table 5-6). Among those genes with duplications, we found statistical significance for the involvement of 11 TSGs in longevity processes (figure 4c; supplementary table 7-8); namely, EEF1A1, H2AFX, HSPD1, MAPK9, GSTP1, PTPN11 (which are known to be involved in ageing); and IGFBP3, NOTCH3, IGFBP3 and SIK1 (known promoters of senescence); while RBX1 and YAP1 are known to inhibit senescence. The results from the enrichment analysis, using the 1,077 TSGs as a reference gene list, infer no significant matches below threshold 
(fdr 0.1), meaning that the biological functions in which those genes with duplications are involved are not significantly overrepresented relative to other TSGs. However, the enrichment analysis made using the human protein-coding genes revealed that they are significantly enriched within several biological processes (figure $4 \mathrm{~b}$, supplementary table 9) such as cell proliferation, cell migration, the cellular apoptosis response to epidermal growth factor stimulus, and metabolic processes. We also report that 17 genes which are duplicated are involved in neurogenesis (e.g. MAPK9, FAT, EFNA5, NOTCH3 and YAP1) or neurological disorders such as the Arnold-Chiari malformation (DKK1, MAF, NOTCH3, SETD2).

In summary, we uncovered an accelerated rate of evolution of TSGs among cetaceans in comparison to other mammalian species. Further, we report 71 gene families with duplications relating to other important anticancer processes, such as DNA repair, metabolism, apoptosis, ageing and senescence. Intriguingly, these have also been reported in other long-lived species.

\section{Discussion}

Cetaceans are the longest-lived mammals and cancer-resistant species. Our comparative analysis revealed that the lineage of cetaceans generally lives longer relative to body mass in comparison to other mammals. We report seven TSGs exhibiting positive selection, one within the ancestor of the group and six within the ancestor of baleen whales, suggesting that baleen whales could have evolved additional anticancer mechanisms. Moreover, the turnover rate of gene gain and loss $(\lambda)$ was accelerated in the cetaceans relative to other mammalian species and we report that 71 TSGs were duplicated in one or more cetacean species. To further understand the evolution of TSGs, we studied the biological processes in which those TSGs exhibiting positive selection and duplications are involved, with a specific focus on important anti-cancer, longevity and senescence pathways. In the ancestor of all cetaceans, we report the signature of positive selection in the $C X C R 2$ gene, known to regulate the recruitment of leukocytes during inflammatory processes. This gene has also been described as a crucial factor in tumour cell dissemination [56,57], immune system disorders such as congenital neutropenia [58], and senescence [59]. In those species that evolved large body masses, replicative senescence is considered to be an important tumour suppressor mechanism [21].

The fact that baleen whales have more positively selected TSGs and a faster gene turnover rate could be interpreted as translating into stronger protective anticancer and ageing 
mechanisms which might have favoured the evolution of gigantism and longevity amongst cetaceans. From those genes exhibiting positive selection in baleen whales, the genes $D A B 2$ and ADAMTS8 have previously been implicated in breast cancer [60,61]; while ADAMTS8 is also involved in angiogenesis and linked to several other forms of cancer, including brain tumours [62], also serving as a promising biomarker in lung neoplasia [63]. On another hand, $A N X A 1$ is an important anti-inflammatory gene related to leukemia and is known to be involved in cellular proliferation, differentiation and apoptosis [64]. Considering the evolution of gigantism in baleen whales, molecular variants involved in angiogenesis, inflammatory processes, and apoptosis may have arisen as a protective mechanism against the development of tumours.

Recent studies of the genome of the humpback whale (Megaptera novaeangliae) also suggested that the evolution of body mass in cetaceans could be related to strong selection in terms of cancer resistance pathways and large segmental duplications [28]. Here we discovered several TSGs exhibiting duplication in cetaceans, an observation that could help future investigations to solve the secrets underlying their longevity and cancer resistance. For example, we found two copies of the MTSS1 gene, implicated in cell proliferation, not only within all cetaceans but also in other several species from the outgroup. Other important genes identified were $R P L 10$, which is involved in the regulation of apoptosis and metabolic processes, and DNMT3A, implicated in neurogenesis and scoliosis, with duplications present within all cetaceans species except for the common dolphin. In fact, in this study, the species with the most gene duplications is the bowhead whale, the only mammal that is recognised as living for more than two centuries. In contrast, the bottlenose dolphin, with fewer duplicated TSGs has the highest incidence of cancer identified within cetaceans $[65,66]$. However, the possibility remains that the differences arising between cancer incidence and longevity within cetaceans may arise due to a reduction in the number of duplicated TSGs (as in the bottlenose dolphin). Moreover, it is also likely that there is a sampling gap which indicates that more studies are required. For example, in the bowhead whale genome, further genes were identified with the signature of positive selection that are known to be involved in DNA repair, cell cycle regulation, resistance to ageing, and cancer [67]. It has been suggested that increasing the number of copies could serve a protective 'guardian' effect in preventing somatic mutations from spreading through the cellular population $[6,68,69]$. It would be interesting to investigate the effect of those conserved TSGs variants identified in whales in human cells. Such an avenue of investigation could eventually lead to an understanding of 
novel pathways that serve to improve the cellular mechanisms protecting against DNA damage, cancer and ageing.

Ageing is the single largest risk factor for the development of cancer and is characterised by the progressive accumulation of cellular damage, yet the mechanisms linking ageing and cancer remain unclear [70]. We report that the turnover rate of TSGs in cetaceans is almost 2.4-fold faster than for other mammals. This acceleration in turnover rate is associated with several TSG duplications, among which eleven genes are known to be associated with longevity and the ageing process. Previous studies have shown that longevity-associated gene families evolve faster (as measured by an increase in the number of gene family members) in long-lived species [71]. One of the main processes which has already been demonstrated to play an important role in ageing and cancer suppression is the irreversible arrest of normally dividing cells, or cell senescence [72]. From those duplicated genes in cetaceans, IGFBP3, SIK1 and NOTCH3 are known senescence promoters. IGFBP3 is a bone health determinant in middle-aged and elderly European men [73] and plays an important in cell proliferation, apoptosis [74], and breast cancer [75]; while SIK1 expression has been correlated with arterial blood pressure, one of the primary pathologies associated with ageing in humans. SIK1 has also been implicated in sympathetic nervous system hyperactivity in mammals [76]. Similarly, the NOTCH3 gene, which has two copies in the Minke whale, also promotes cell senescence and has been correlated with the development of brain lesions with the age [77]. The increase in the number of copies of ageing genes in cetaceans could provide additional protection against the development of several types of cancer as well as ageing-related diseases.

Although in this study we primarily focus on the roles of genes in cancer and ageing, it is important to highlight that they could also perform functions related to other phenotypic outcomes such as those associated with phenotypic remodelling in response to the conquest of the aquatic environment. For example, the DSC 3 gene, positively selected in Mysticeti, is a biomarker for colorectal [78] and breast cancer [79]. However, mutations in this gene are also correlated with hair loss disorders [80] such as hypotrichosis [81]. Hair loss in cetaceans was related to their hydrodynamic adaptation in an aquatic environment [82]. In the same vein, several TSGs which have been duplicated in whales (e.g. ACVR1C, CTBP1, DDX3X) are involved in metabolic processes that may have responded to those selective pressures involved in energy consumption, diet, and increases in body mass. Furthermore, $A N X A 1$, an anti-inflammatory gene, and EPHA2, an antibody receptor target in chemotherapy [83], are also involved in blood vessel development, another important feature in underwater evolution 
[84]. Considering the pleiotropic effects of TSGs, a more comprehensive understanding of the consequences of natural selection at a molecular level in the evolutionary history of cetaceans is required.

\section{Conclusions}

The main goal of this study was to shed light on the evolution of TSGs in cetaceans. We report signals of positive selection in 7 TSGs and an accelerated gene turnover rate in comparison to other mammals, with duplications arising in 71 genes in one or more cetacean species. Those genes with the characteristic signature of positive selection are implicated in multiple diseases, including bladder cancer, breast cancer, leukaemia and immune system disorders (e.g. congenital neutropenia). The turnover rate of TSGs was almost 2.4 times higher in cetaceans as compared to other mammals. From those genes with duplications, 11 are linked to longevity and senescence (e.g. NOTCH3, IGFBP3, and SIK1). Although the enrichment analysis using the 1077 TSGs as a background did not lead to significantly enriched biological categories, the same analysis using all protein coding genes as a background gives us a sense of which functions are associated with the duplicated genes. Some of those functions included cell proliferation, apoptosis, and metabolism. Remarkably, most of the genes exhibiting evidence of positive selection and duplication were found amongst the baleen whales. Considering that the clade of baleen whales includes the biggest extant species known and that such species are also among the longest living of all mammals, we suggest that they could have evolved with additional mechanisms that conferred both resistance to cancer and longevity. Nonetheless, due to possible pleiotropic effects of TSGs, the genes we identified as duplicated and under positive selection could also have been important in the cetacean evolution to aquatic life. Studying genes that are related to human malignancies from a comparative perspective offers a novel avenue that could provide additional clues about the role of natural selection in the evolution of all species and, potentially, lead to the discovery of novel therapeutic approaches. This study provides evolutionary evidence that natural selection among TSGs could act favourably for those species with larger body sizes and greatly extended life spans, thereby providing insights into the genetic basis associated with the evolution of disease resistance. We also highlight the importance of cetaceans as an evolutionary model to understand the genesis of ageing disorders in humans and other animals. 


\section{Data accessibility}

All data generated or analysed during this study are included in this published article and in the supplementary information. The supplementary files 1 and 2 are deposited in the Dryad repository.

\section{Authors' contributions}

D.T.M and J.C.O conceived the project; D.T.M performed the bioinformatic analysis. D.T.M wrote the manuscript; J.C.O and J.P.M edited the manuscript and were the project advisors.

\section{Competing interests}

We declare we have no competing interests.

\section{Funding}

Comisión Nacional de Investigación Científica y Tecnológica (CONICYT) - Chile through the doctoral scholarship $\mathrm{N}^{\circ} 21170433$ and the scholarship from MECESUP AUS2003 to DTM. Fondo Nacional de Desarrollo Científico y Tecnológico (FONDECYT) grant 1160627 and Millennium Nucleus of Ion Channels Associated Diseases (MiNICAD), Iniciativa Científica Milenio, Ministry of Economy, Development and Tourism, Chile to JCO. AnAge is supported by funding from the Biotechnology and Biological Sciences Research Council (BB/R014949/1) to JPM.

\section{Acknowledgements}

Members of the Integrative Genomics of Ageing Group at the University of Liverpool, for all the support and advice during DTM's internship. To Richard Gregory in the Centre for Genomic Research (CGR) and Ian C. Smith in the Advanced Research Computing at the University of Liverpool for their help with computing resources. To Dr. Andres Parada, for the academic discussions and with programming advice. To Dimar Gonzales for the programming advice. To Alejandra Tejada-Martinez for the graphic designs. To Dr. Priyanka Raina for the grammatical revision of the manuscript. Finally to Jesus Tejada and Gloria Martinez for their unconditional support. 


\section{References}

1. Peto R. 1977 Origins of Human Cancer. (eds Hiatt, H. H., Watson, J. D. \& Winsten, J. A.). Cold Spring Harbor Publications, 1977.

2. Nunney L. 1999 Lineage selection and the evolution of multistage carcinogenesis. Proceedings of the Royal Society of London. Series B: Biological Sciences. 266, 493-498.

3. Peto R. 2015 Quantitative implications of the approximate irrelevance of mammalian body size and lifespan to lifelong cancer risk. Philos. Trans. R. Soc. Lond. B Biol. Sci. 370.

4. Tollis M, Boddy AM, Maley CC. 2017 Peto's Paradox: how has evolution solved the problem of cancer prevention? BMC Biol. 15, 60 .

5. Tomasetti C, Durrett R, Kimmel M, Lambert A, Parmigiani G, Zauber A, Vogelstein B. 2017 Role of stem-cell divisions in cancer risk. Nature 548, E13-E14.

6. Caulin AF, Maley CC. 2011 Peto's Paradox: evolution's prescription for cancer prevention. Trends Ecol. Evol. 26, 175-182.

7. Green J, Cairns BJ, Casabonne D, Lucy Wright F, Reeves G, Beral V. 2011 Height and cancer incidence in the Million Women Study: prospective cohort, and meta-analysis of prospective studies of height and total cancer risk. The Lancet Oncology. 12, 785-794.

8. Nagy JD, Victor EM, Cropper JH. 2007 Why don't all whales have cancer? A novel hypothesis resolving Peto's paradox. Integrative and Comparative Biology. 47, 317-328.

9. Aktipis CA, Boddy AM, Jansen G, Hibner U, Hochberg ME, Maley CC, Wilkinson GS. 2015 Cancer across the tree of life: cooperation and cheating in multicellularity. Philos. Trans. R. Soc. Lond. B Biol. Sci. 370.

10. Roche B, Hochberg ME, Caulin AF, Maley CC, Gatenby RA, Misse D, Thomas F. 2012 Natural resistance to cancers: a Darwinian hypothesis to explain Peto's paradox. BMC Cancer. 12.

11. Nunney L, Muir B. 2015 Peto's paradox and the hallmarks of cancer: constructing an evolutionary framework for understanding the incidence of cancer. Philosophical Transactions of the Royal Society B: Biological Sciences. 370, 20150161.

12. DeGregori J. 2017 Connecting cancer to its causes requires incorporation of effects on tissue microenvironments. Cancer Res. 77, 6065.

13. Boddy AM, Harrison TM, Abegglen LM. 2020 Comparative Oncology: New Insights into an Ancient Disease. iScience 23, 101373.

14. Nunney L, Maley CC, Breen M, Hochberg ME, Schiffman JD. 2015 Peto's paradox and the promise of comparative oncology. Philosophical Transactions of the Royal Society B: Biological Sciences. 370, 20140177.

15. Tollis M, Schiffman JD, Boddy AM. 2017 Evolution of cancer suppression as revealed 
by mammalian comparative genomics. Curr. Opin. Genet. Dev. 42, 40-47.

16. Wensink MJ. 2016 Size, longevity and cancer: age structure. Proc. Biol. Sci. 283.

17. Tacutu R et al. 2018 Human Ageing Genomic Resources: new and updated databases. Nucleic Acids Res. 46, D1083-D1090.

18. Ruby JG, Smith M, Buffenstein R. 2018 Naked Mole-Rat mortality rates defy gompertzian laws by not increasing with age. Elife 7.

19. Liang S, Mele J, Wu Y, Buffenstein R, Hornsby PJ. 2010 Resistance to experimental tumourigenesis in cells of a long-lived mammal, the naked mole-rat (Heterocephalus glaber). Aging Cell. 9, 626-635.

20. Seluanov A, Gladyshev VN, Vijg J, Gorbunova V. 2018 Mechanisms of cancer resistance in long-lived mammals. Nature Reviews Cancer. 18, 433-441.

21. Tian X, Doerig K, Park R, Can Ran Qin A, Hwang C, Neary A, Gilbert M, Seluanov A, Gorbunova V. 2018 Evolution of telomere maintenance and tumour suppressor mechanisms across mammals. Philos. Trans. R. Soc. Lond. B Biol. Sci. 373.

22. Foley NM et al. 2018 Growing old, yet staying young: The role of telomeres in bats' exceptional longevity. Sci Adv 4, eaao0926.

23. Zhang $\mathrm{G}$ et al. 2013 Comparative analysis of bat genomes provides insight into the evolution of flight and immunity. Science 339, 456-460.

24. Cailliet GM, Andrews AH, Burton EJ, Watters DL, Kline DE, Ferry-Graham LA. 2001 Age determination and validation studies of marine fishes: do deep-dwellers live longer? Experimental Gerontology. 36, 739-764.

25. Sulak M, Fong L, Mika K, Chigurupati S, Yon L, Mongan NP, Emes RD, Lynch VJ. 2016 TP53 copy number expansion is associated with the evolution of increased body size and an enhanced DNA damage response in elephants. eLife. 5.

26. Mancia A. 2018 On the revolution of cetacean evolution. Mar. Genomics 41, 1-5.

27. Sun Y, Liu Y, Sun X, Lin Y, Yin D, Xu S, Yang G. 2019 Insights into body size variation in cetaceans from the evolution of body-size-related genes. BMC Evol. Biol. $19,157$.

28. Tollis M et al. 2019 Return to the Sea, Get Huge, Beat Cancer: An Analysis of Cetacean Genomes Including an Assembly for the Humpback Whale (Megaptera novaeangliae). Mol. Biol. Evol. 36, 1746-1763.

29. Tian X, Seluanov A, Gorbunova V. 2017 Molecular Mechanisms Determining Lifespan in Short- and Long-Lived Species. Trends Endocrinol. Metab. 28, 722-734.

30. Magalhães JP de, de Magalhães JP, Kean M. 2013 Endless paces of degeneration-applying comparative genomics to study evolution's moulding of longevity. EMBO reports. 14, 661-662. 
31. Nunney L. 2013 The real war on cancer: the evolutionary dynamics of cancer suppression. Evol. Appl. 6, 11-19.

32. Gorbunova V, Seluanov A, Zhang Z, Gladyshev VN, Vijg J. 2014 Comparative genetics of longevity and cancer: insights from long-lived rodents. Nat. Rev. Genet. 15, 531-540.

33. David Orme, Rob Freckleton, Gavin Thomas, Thomas Petzoldt, Susanne Fritz, Nick Isaac, Will Pearse. 2012 caper: Comparative Analyses of Phylogenetics and Evolution in R.

34. Uyeda JC, Pennell MW, Miller ET, Maia R, McClain CR. 2017 The Evolution of Energetic Scaling across the Vertebrate Tree of Life. Am. Nat. 190, 185-199.

35. Pruitt KD, Tatusova T, Maglott DR. 2007 NCBI reference sequences (RefSeq): a curated non-redundant sequence database of genomes, transcripts and proteins. Nucleic Acids Research. 35, D61-D65.

36. Huang Y, Niu B, Gao Y, Fu L, Li W. 2010 CD-HIT Suite: a web server for clustering and comparing biological sequences. Bioinformatics. 26, 680-682.

37. Altenhoff AM et al. 2019 OMA standalone: orthology inference among public and custom genomes and transcriptomes. Genome Res. 29, 1152-1163.

38. Train C-M, Glover NM, Gonnet GH, Altenhoff AM, Dessimoz C. 2017 Orthologous Matrix (OMA) algorithm 2.0: more robust to asymmetric evolutionary rates and more scalable hierarchical orthologous group inference. Bioinformatics. 33, i75-i82.

39. Train C-M, Pignatelli M, Altenhoff A, Dessimoz C. 2019 iHam and pyHam: visualizing and processing hierarchical orthologous groups. Bioinformatics 35, 2504-2506.

40. Katoh K, Standley DM. 2013 MAFFT Multiple Sequence Alignment Software Version 7: Improvements in Performance and Usability. Molecular Biology and Evolution. 30, $772-780$.

41. Brown JW, Walker JF, Smith SA. 2017 Phyx: phylogenetic tools for unix. Bioinformatics. 33, 1886-1888.

42. Hubisz MJ, Pollard KS, Siepel A. 2011 PHAST and RPHAST: phylogenetic analysis with space/time models. Briefings in Bioinformatics. 12, 41-51.

43. Yang Z. 2007 PAML 4: Phylogenetic Analysis by Maximum Likelihood. Molecular Biology and Evolution. 24, 1586-1591.

44. Huerta-Cepas J, Serra F, Bork P. 2016 ETE 3: Reconstruction, Analysis, and Visualization of Phylogenomic Data. Molecular Biology and Evolution. 33, 1635-1638.

45. Zhang J. 2005 Evaluation of an Improved Branch-Site Likelihood Method for Detecting Positive Selection at the Molecular Level. Molecular Biology and Evolution. 22, 2472-2479.

46. Benjamini Y, Hochberg Y. 1995 Controlling the False Discovery Rate: A Practical and Powerful Approach to Multiple Testing. Journal of the Royal Statistical Society: Series 
B (Methodological). 57, 289-300.

47. Han MV, Thomas GWC, Lugo-Martinez J, Hahn MW. 2013 Estimating Gene Gain and Loss Rates in the Presence of Error in Genome Assembly and Annotation Using CAFE 3. Molecular Biology and Evolution. 30, 1987-1997.

48. Kumar S, Stecher G, Suleski M, Blair Hedges S. 2017 TimeTree: A Resource for Timelines, Timetrees, and Divergence Times. Molecular Biology and Evolution. 34, 1812-1819.

49. Revell LJ. 2012 phytools: an R package for phylogenetic comparative biology (and other things). Methods in Ecology and Evolution. 3, 217-223.

50. In press. Website. See Team, R. Core. A language and environment for statistical computing. Vienna, Austria: R Foundation for Statistical Computing 2012." URL https://www.R-project.org. (2019) (accessed on 12 October 2020).

51. Zhang X, Kong L, Gao Y, Ma H. 2018 Odontoceti phylogeny and divergence data resolved: evidence from nuclear genes and complete mitochondrial genomes of Neophocaena phocaenoides. Conservation Genetics Resources. 10, 47-49.

52. Liao Y, Wang J, Jaehnig EJ, Shi Z, Zhang B. 2019 WebGestalt 2019: gene set analysis toolkit with revamped UIs and APIs. Nucleic Acids Res. 47, W199-W205.

53. Khatri P, Sirota M, Butte AJ. 2012 Ten years of pathway analysis: current approaches and outstanding challenges. PLoS Comput. Biol. 8, e1002375.

54. Avelar RA et al. 2020 A multidimensional systems biology analysis of cellular senescence in aging and disease. Genome Biology. 21.

55. Shen L. 2014 GeneOverlap: An R package to test and visualize gene overlaps. $\mathrm{R}$ Package

56. Acosta JC, Gil J. 2009 A Role for CXCR2 in Senescence, but What about in Cancer? Cancer Research. 69, 2167-2170.

57. Acosta JC, Gil J. 2012 Senescence: a new weapon for cancer therapy. Trends in Cell Biology. 22, 211-219.

58. Inserm, INSERM. 2020 Autosomal recessive severe congenital neutropenia due to CXCR2 deficiency. Definitions.

59. Acosta JC et al. 2008 Chemokine Signaling via the CXCR2 Receptor Reinforces Senescence. Cell. 133, 1006-1018.

60. Tian X, Zhang Z. 2018 miR-191/DAB2 axis regulates the tumourigenicity of estrogen receptor-positive breast cancer. IUBMB Life 70, 71-80.

61. Porter S et al. 2006 ADAMTS8 and ADAMTS15 expression predicts survival in human breast carcinoma. International Journal of Cancer. 118, 1241-1247.

62. Dunn JR, Reed JE, du Plessis DG, Shaw EJ, Reeves P, Gee AL, Warnke P, Walker C. 
2006 Expression of ADAMTS-8, a secreted protease with antiangiogenic properties, is downregulated in brain tumours. British Journal of Cancer. 94, 1186-1193.

63. Zhou D, Tang W, Liu X, An H-X, Zhang Y. 2017 Clinical verification of plasma messenger RNA as novel noninvasive biomarker identified through bioinformatics analysis for lung cancer. Oncotarget 8, 43978-43989.

64. Guo C, Liu S, Sun M-Z. 2013 Potential role of Anxa1 in cancer. Future Oncology. 9, 1773-1793.

65. Bossart GD, Ewing R, Herron AJ, Cray C, Mase B, Decker SJ, Alexander JW, Altman NH. 1997 Immunoblastic Malignant Lymphoma in Dolphins: Histologic, Ultrastructural, and Immunohistochemical Features. Journal of Veterinary Diagnostic Investigation. 9, 454-458.

66. Newman SJ, Smith SA. 2006 Marine Mammal Neoplasia: A Review. Veterinary Pathology. 43, 865-880.

67. Keane $\mathrm{M}$ et al. 2015 Insights into the evolution of longevity from the bowhead whale genome. Cell Rep. 10, 112-122.

68. García-Cao I, García-Cao M, Martín-Caballero J, Criado LM, Klatt P, Flores JM, Weill J-C, Blasco MA, Serrano M. 2002 'Super p53' mice exhibit enhanced DNA damage response, are tumor resistant and age normally. EMBO J. 21, 6225-6235.

69. Vazquez JM, Sulak M, Chigurupati S, Lynch VJ. 2018 A Zombie LIF Gene in Elephants Is Upregulated by TP53 to Induce Apoptosis in Response to DNA Damage. Cell Rep. 24, 1765-1776.

70. Chatsirisupachai K, Palmer D, Ferreira S, de Magalhães JP. 2019 A human tissue-specific transcriptomic analysis reveals a complex relationship between aging, cancer, and cellular senescence. Aging Cell 18, e13041.

71. Li Y, de Magalhães JP. 2013 Accelerated protein evolution analysis reveals genes and pathways associated with the evolution of mammalian longevity. Age 35, 301-314.

72. Magalhães JP de, de Magalhães JP, Passos JF. 2018 Stress, cell senescence and organismal ageing. Mechanisms of Ageing and Development. 170, 2-9.

73. Pye SR et al. 2011 Influence of Insulin-Like Growth Factor Binding Protein (IGFBP)-1 and IGFBP-3 on Bone Health: Results from the European Male Ageing Study. Calcified Tissue International. 88, 503-510.

74. Pollak MN, Schernhammer ES, Hankinson SE. 2004 Insulin-like growth factors and neoplasia. Nature Reviews Cancer. 4, 505-518.

75. Burger AM, Leyland-Jones B, Banerjee K, Spyropoulos DD, Seth AK. 2005 Essential roles of IGFBP-3 and IGFBP-rP1 in breast cancer. Eur. J. Cancer 41, 1515-1527.

76. Pires NM et al. 2019 Acute salt loading induces sympathetic nervous system overdrive in mice lacking salt-inducible kinase 1 (SIK1). Hypertens. Res. 42, 1114-1124. 
77. Schmidt $\mathrm{H}$ et al. 2011 Genetic variants of the NOTCH3 gene in the elderly and magnetic resonance imaging correlates of age-related cerebral small vessel disease. Brain. 134, 3384-3397.

78. Cui T, Chen Y, Yang L, Knösel T, Zöller K, Huber O, Petersen I. 2011 DSC3 expression is regulated by $\mathrm{p} 53$, and methylation of DSC3 DNA is a prognostic marker in human colorectal cancer. British Journal of Cancer. 104, 1013-1019.

79. Oshiro MM et al. 2005 Epigenetic silencing of DSC3 is a common event in human breast cancer. Breast Cancer Res. 7, R669-80.

80. Lee JYW, McGrath JA. 2020 Mutations in genes encoding desmosomal proteins: spectrum of cutaneous and extracutaneous abnormalities. British Journal of Dermatology.

81. Ayub M, Basit S, Jelani M, Rehman FU, Iqbal M, Yasinzai M, Ahmad W. 2009 A Homozygous Nonsense Mutation in the Human Desmocollin-3 (DSC3) Gene Underlies Hereditary Hypotrichosis and Recurrent Skin Vesicles. The American Journal of Human Genetics. 85, 515-520.

82. Nery MF, González DJ, Opazo JC. 2013 How to Make a Dolphin: Molecular Signature of Positive Selection in Cetacean Genome. PLoS One 8, e65491.

83. Tandon M, Vemula SV, Mittal SK. 2011 Emerging strategies for EphA2 receptor targeting for cancer therapeutics. Expert Opin. Ther. Targets 15, 31-51.

84. Huelsmann M, Hecker N, Springer MS, Gatesy J, Sharma V, Hiller M. 2019 Genes lost during the transition from land to water in cetaceans highlight genomic changes associated with aquatic adaptations. Sci Adv 5, eaaw6671. 


\section{Tables}

Table 1. Human diseases related to genes with a signature of positive selection in the ancestor of cetaceans and baleen whales. The information was obtained from GeneCards (https://www.genecards.org/),

\begin{tabular}{|c|c|c|}
\hline Branch & & Diseases associated \\
\hline $\begin{array}{l}\text { Ancestor } \\
\text { Cetacea }\end{array}$ & CXCR2 & $\begin{array}{l}\text { Autosomal recessive severe congenital } \\
\text { neutropenia - Neutrophil migration - } \\
\text { Immune system }\end{array}$ \\
\hline \multirow{6}{*}{$\begin{array}{l}\text { Ancestor } \\
\text { Mysticeti }\end{array}$} & ADAMTS8 & $\begin{array}{l}\text { Diseases of glycosylation - Lung } \\
\text { neoplasm }\end{array}$ \\
\hline & ANXA1 & $\begin{array}{l}\text { Anti-inflammatory activity. Hairy Cell } \\
\text { Leukemia and Penis Squamous Cell } \\
\text { Carcinoma. }\end{array}$ \\
\hline & DAB2 & $\begin{array}{l}\text { Malignant epithelial mesothelioma - } \\
\text { Hypercholesterolemia autosomal } \\
\text { recessive - ovarian carcinoma }\end{array}$ \\
\hline & DSC3 & $\begin{array}{l}\text { Hypotrichosis - Recurrent skin vesicles - } \\
\text { Subcorneal pustular dermatosis }\end{array}$ \\
\hline & EPHA2 & $\begin{array}{l}\text { Cataract } 6 \text {, multiple types - Early-Onset } \\
\text { posterior subcapsular cataract }\end{array}$ \\
\hline & TMPRSS11A & $\begin{array}{l}\text { Iron-Refractory iron deficiency anemia - } \\
\text { Ichthyosis follicular }\end{array}$ \\
\hline
\end{tabular}




\section{Figures}

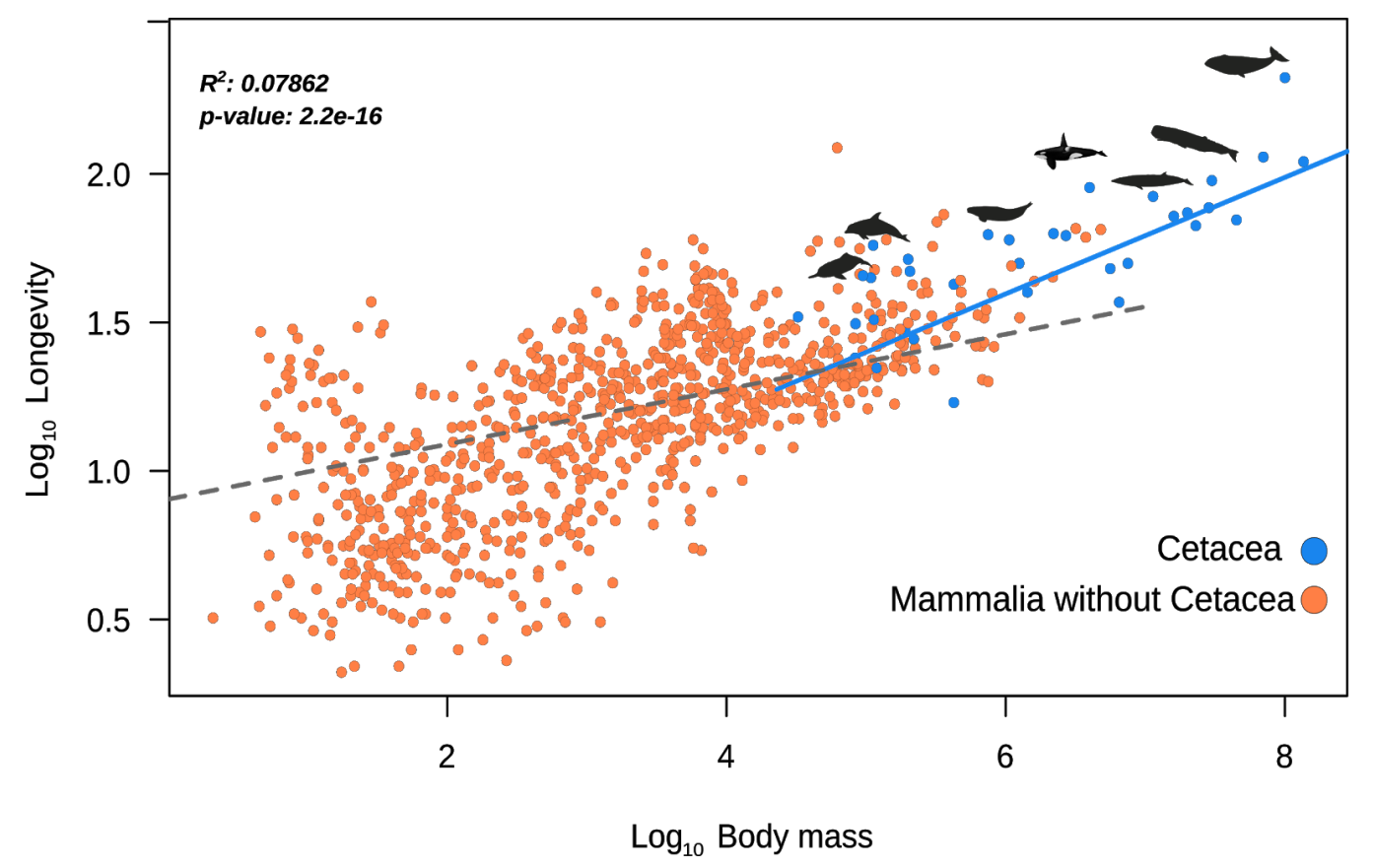

Figure 1. Phylogenetic generalized least squares (PGLS) between the body mass and longevity across 930 mammalian species. The results from the regression showed that the relationship between body mass and longevity is significantly different in cetaceans as compared to other mammals $(p<0.001)$. 
a)

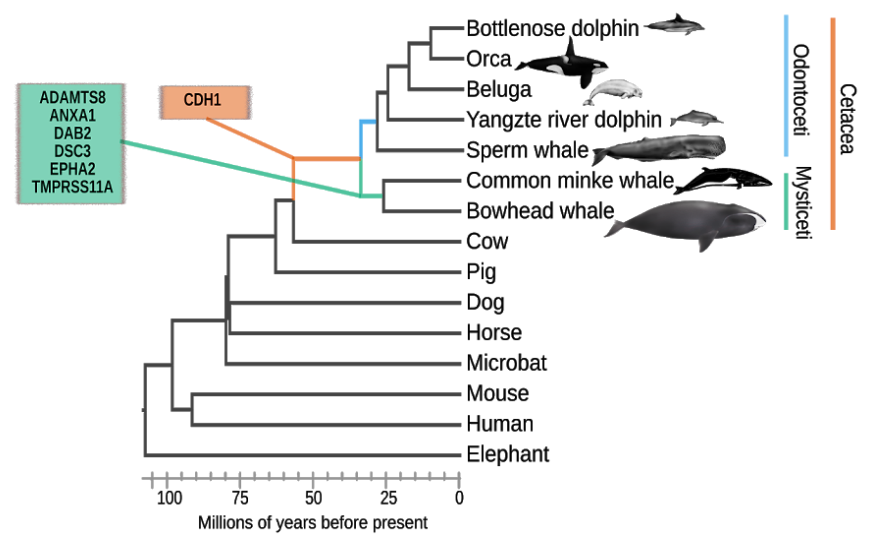

b)

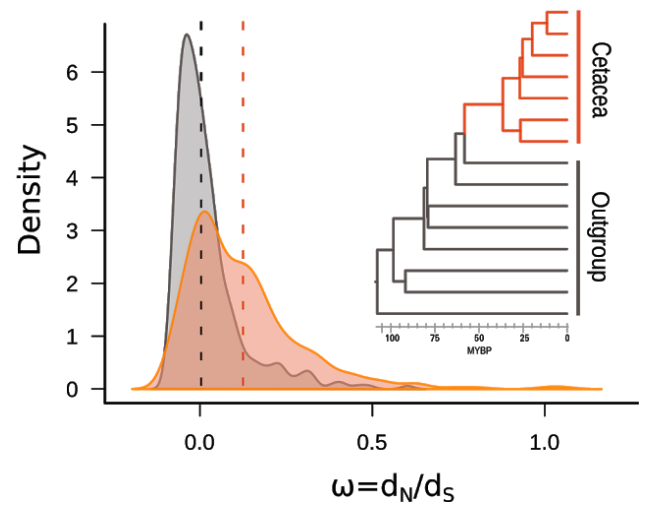

Figure 2. Codon-based models of evolution ( $\omega=\mathrm{dN} / \mathrm{dS}$ models). a) Tumour suppressor genes with the signature of positive selection in different branches of the cetacean tree of life. $b$ ) Distribution of the omega values estimated separately for cetaceans and all other branches of the tree. The height of the curves is relative to the number of genes with the observed omega values and the dotted lines represent the mean value for cetaceans (orange) and the outgroup (grey). The Kolmogorov-Smirnov test showed that the distributions have statistically significant differences ( $p$-value $<0.01)$. 

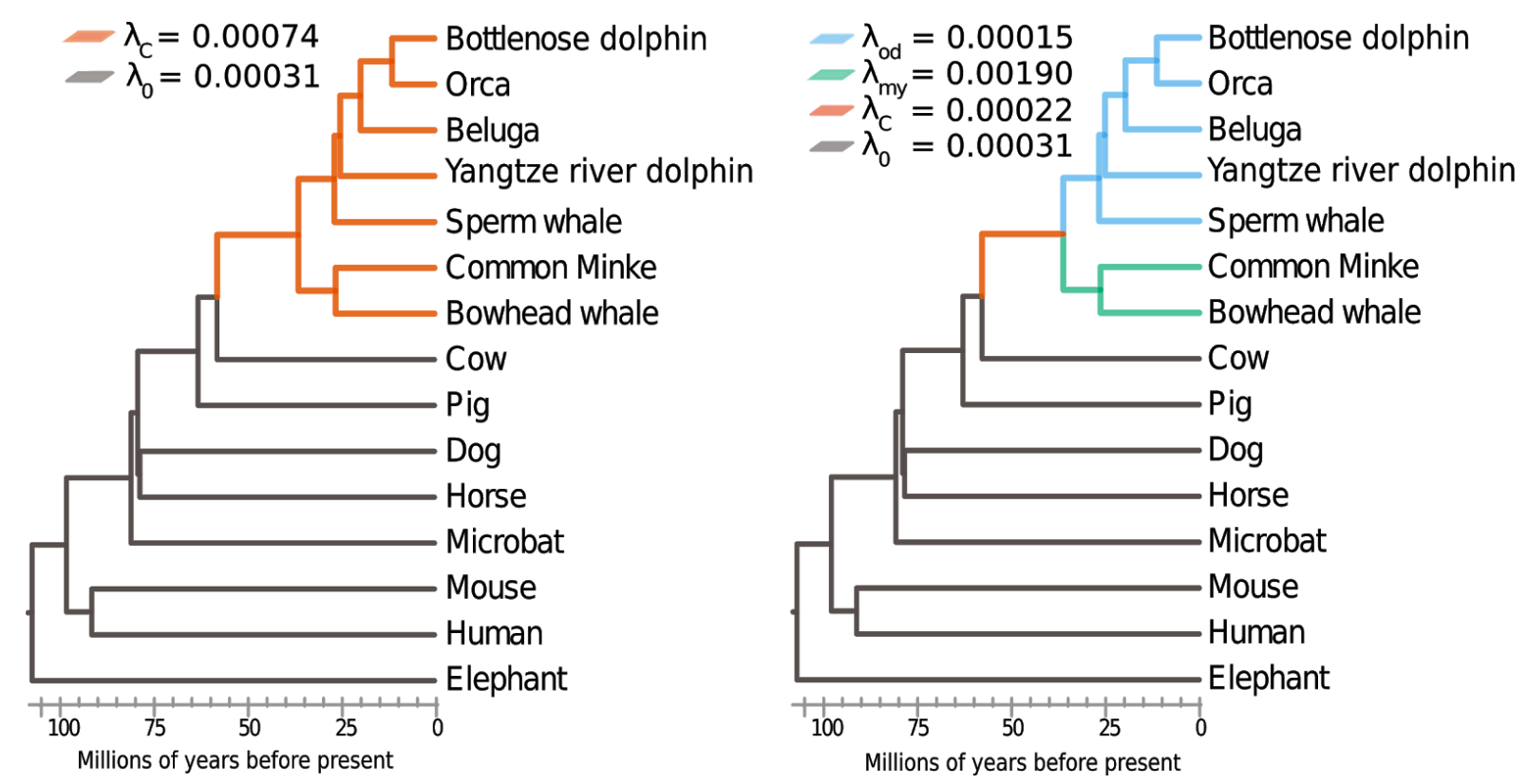

Figure 3. Turnover rates of tumour suppressor genes (TSGs) in cetaceans. a) The first model represents the rate of evolution ( $\lambda$ ) of TSGs for the total group of cetacea (orange) and the rate of evolution of TSGs for the outgroup (grey). The $\lambda$ values show that the gene turnover rate of TSGs in cetaceans is almost 2.4 times faster than for other placental mammals. b) In the second model, the $\lambda$ values represent the rate of evolution for the total group of baleen whales (green), the total group of toothed whales (light blue), and for the ancestor of all cetaceans (orange). The gene turnover rate in the total group of baleen whales is faster in relation with other groups. 
a)

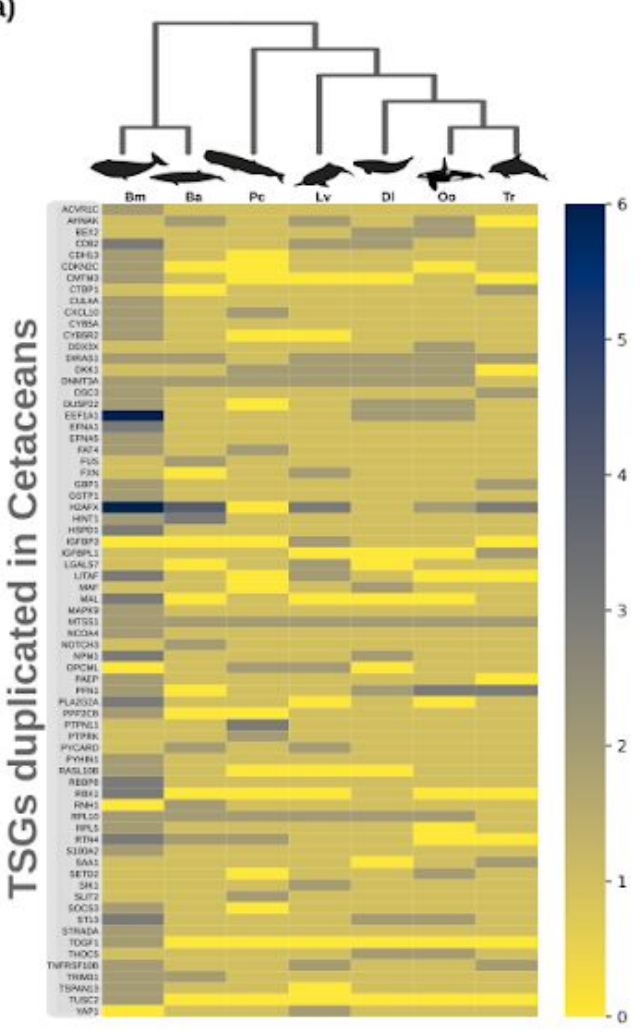

b)

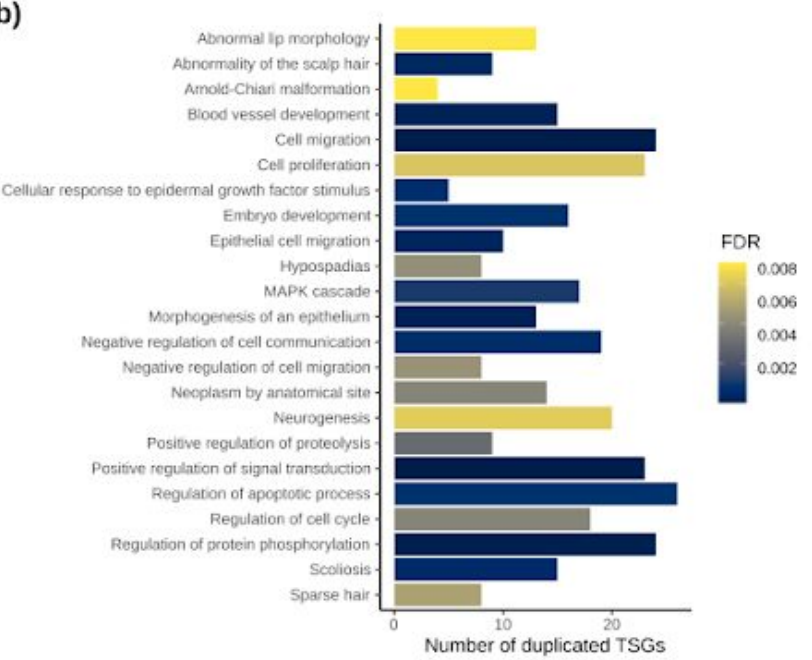

c)

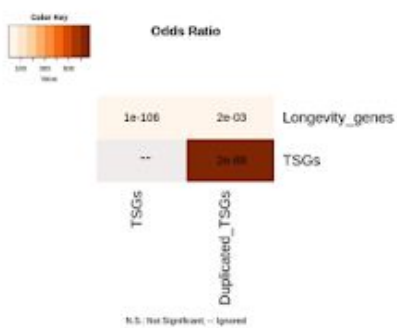

Figure 4. Tumour suppressor genes (TSGs) with duplications in cetaceans. a) Heat map showing copy number variation of TSGs in cetaceans. The colour code corresponds to the number of copies. Key: Tt -Tursiops truncatus, Oc-Orcinus Orca, Dl-Delphinapterus leucas, Lv-Lipotes vexillifer, Pc-Physeter catodon (synonym name of Physeter macrocephalus), Ba-Balaenoptera acutorostrata and Bm-Balaena mysticetus. b) Enrichment analysis for the duplicated genes found in cetaceans using the whole genome as background. The image shows the first 30 categories with an FDR $<0.01$ for biological processes, Diseases OMIM, and Human phenotype ontology. c) Overlap analysis showing that those TSGs with duplications are involved in the longevity process more than would be expected by chance (adjusted p-value $<0.01$ ). 